\title{
Phase-flip chimera induced by environmental nonlocal coupling
}

\author{
V. K. Chandrasekar ${ }^{1}$, R. Gopal ${ }^{2,3}$, D. V. Senthilkumar ${ }^{4}$, and M. Lakshmanan ${ }^{2}$ \\ ${ }^{1}$ Centre for Nonlinear Science \& Engineering, School of Electrical \& Electronics Engineering, \\ SASTRA University, Thanjavur- 613 401, India. \\ ${ }^{2}$ Centre for Nonlinear Dynamics, School of Physics, \\ Bharathidasan University, Tiruchirapalli-620024, India. \\ ${ }^{3}$ Department of Physics, Nehru Memorial College, Puthanampatti, Tiruchirapalli 621 007, India. \\ 4 School of Physics, Indian Institute of Science Education and Research, Thiruvananthapuram-695016, India.
}

(Dated: October 1, 2018)

\begin{abstract}
We report the emergence of a collective dynamical state, namely phase-flip chimera, from an ensemble of identical nonlinear oscillators that are coupled indirectly via the dynamical variables from a common environment, which in turn are nonlocally coupled. The phase-flip chimera is characterized by the coexistence of two adjacent out-of-phase synchronized coherent domains interspersed by an incoherent domain, in which the nearby oscillators are in out-of-phase synchronized states. Attractors of the coherent domains are either from the same or different basins of attractions depending on whether they are periodic or chaotic. Conventional chimera precedes the phase-flip chimera in general. Further, the phase-flip chimera emerges after the completely synchronized evolution of the ensemble in contrast to conventional chimeras which emerge as an intermediate state between completely incoherent and coherent states. We have also characterized the observed dynamical transitions using the strength of incoherence, probability distribution of correlation coefficient and the framework of master stability function.
\end{abstract}

PACS numbers: 05.45.-a, 05.45.Xt, 89.75.-k

\section{INTRODUCTION}

Identification of an intriguing collective dynamical state, namely the chimera state, in an ensemble of coupled identical nonlinear oscillators with nonlocal coupling [1-4] has initiated intense research activities in the recent literature [4 9]. A chimera state represents a spatially inhomogeneous state characterized by coexisting coherent and incoherent domains in an ensemble of identical oscillators. Experimentally, chimera has also been revealed in populations of coupled chemical oscillators [10], in electro-optical systems [11] and in metronomes [12]. Real world examples mimicking chimera states can be found in power grids [13], in unihemispheric sleep of animals 14, in multiple time scales of sleep dynamics [15], etc. Different types of chimera states such as amplitude mediated chimera [16], intensity induced chimera 17], and so on have also been identified [18]. Recently, it has been shown that a symmetry breaking coupling in the Stuart-Landau oscillators leads to the manifestation of chimera death [19, 20]. The collective state with inhomogeneous flipping between the steady states (oscillation death) of an ensemble was called chimera death. Very recently, noise induced coherenceresonance chimeras in a network of excitable elements was reported [21]. Coherence-resonance chimeras are associated with alternating switching of the location of coherent and incoherent domains.

In this manuscript, we unravel a novel dynamical regime emerging from the inhomogeneous synchronized states. In particular, we consider an ensemble of identical nonlinear oscillators coupled via a common dynamic environment with nonlocal coupling. We show that the ensemble of oscillators splits into coexisting coherent and incoherent domains for appropriate strength of the nonlocal coupling. The nearby oscillators in the coherent domains exhibit in-phase synchronized oscillations while the adjacent oscillators in the incoherent domains exhibit out-of-phase oscillations. It is to be noted that the term coherent/incoherent domains here represents the homogenous/inhomogenous nature of the dynamics of the attractors in the corresponding domain and does not refer to the nature of the attractors, that is whether they are coherent attractors with a fixed center of rotation or incoherent attractors with more than one center of rotation.

Further, we find that some of the nearby coherent domains exhibit in-phase oscillations in one of the domains and anti-phase oscillations in the other resembling the phase-flip bifurcation/transition 22]. Specifically, the out-of-phase synchronized nearby coherent domains are interspersed by an incoherent domain (where the phases of the adjacent oscillators flip between 0 and $\pi$ ) at the phase-flip transition, which we call a phase-flip chimera. It is a dynamically active emerging behavior in contrast to the chimera death [19, 20], where nearby oscillators populate the same branch of the inhomogeneous steady state in the coherent domain while the nearby oscillators populate different branches of the inhomogeneous steady state in the incoherent domain. Further, we find that the conventional chimera is preceded by the phaseflip chimera in addition to the other collective dynamical regimes such as coherent and synchronized states in an ensemble of the paradigmatic Rössler oscillators both in the periodic and chaotic regimes. We have used the measures, namely the strength of incoherence and the probability distribution of the correlation coefficient to characterize the phase-flip chimeras and the observed dy- 

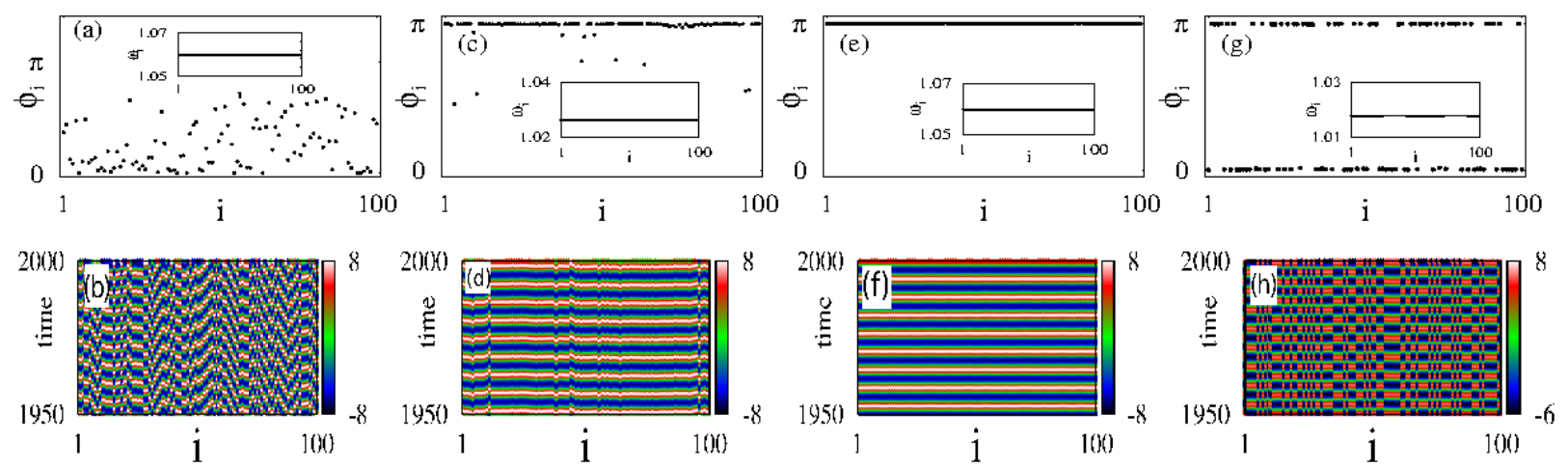

FIG. 1. (Color online) Snapshots of the instantaneous phases $\phi_{i}$ (top row) and the space-time evolution (bottom row) of the ensemble of Rössler oscillators in the periodic regime for the coupling radius $r=0.3$ of the nonlocal coupling and for different values of the strength of the nonlocal coupling exhibiting (a)-(b) desynchronized state for $\varepsilon=0.02$, (c)-(d) chimera state for $\varepsilon=0.08$, (e)-(f) synchronized state for $\varepsilon=0.5$ and $(\mathrm{g})-(\mathrm{h})$ phase-flip chimera for $\varepsilon=1.5$. Time averaged frequencies of all the oscillators are shown in the insets. f Other parameter values are $a=0.165, b=0.4, c=8.5$ and $\alpha=1$.

namical transitions. In addition, we have also employed the framework of the master-stability function (MSF) to demarcate the synchronized and desynchronized parameter space which agrees very well with the simulation results. It is to be pointed out that the synchronized regime is a multistability regime coexisting with conventional chimeras and phase-flip chimeras depending on the distribution of the initial conditions.

The plan of the paper is as follows. We discuss the emergence of phase-flip chimera in an ensemble of Rössler oscillators in the periodic regime in Sec. II and in the chaotic regime in Sec. III. We provide certain quantification measures such as the strength of incoherence, probability distribution of the correlation coefficient and the framework of the master stability functon to characterize the different collective dynamical behavior in the ensemble of oscillators in Sec. IV. Discussion on the global dynamical behavior in terms of two-parameter phase diagrams is provided in Sec. V. Emergence of phase-flip chimera with global coupling among the agents in the common environment will be discussed in Sec. VI and conclusion will be provided in Sec. VII. In Appendix A, we consider the effect of relaxation time while the effect of coupling to other variables is discussed in Appendix B.

\section{PHASE-FLIP CHIMERA IN AN ENSEMBLE OF RÖSSLER OSCILLATORS IN THE PERIODIC REGIME}

To elucidate the above results, we consider an ensemble of identical Rössler oscillators with a common dynamic environmental coupling represented as

$$
\begin{aligned}
\dot{x}_{i} & =-y_{i}-z_{i}, \\
\dot{y}_{i} & =x_{i}+a y_{i} \\
\dot{z}_{i} & =b+z_{i}\left(x_{i}-c\right)+k w_{i}, \\
\dot{w}_{i} & =-\alpha w_{i}+\frac{z_{i}}{2}+\frac{\varepsilon}{2 P} \sum_{j=i-P}^{j=i+P}\left(w_{j}-w_{i}\right),
\end{aligned}
$$

$i=1, \ldots, N$, where $a, b, c$ and $\alpha$ are the system parameters, $N$ is the number of oscillators in the ensemble. The oscillators in the ensemble are coupled indirectly via nonlocally coupled dynamic agents $w_{i}$ in the common environment. $k$ is the strength with which the agent $w_{i}$ from the common environment interacts with the $i$ th oscillator in the ensemble. $\varepsilon$ is the strength of the nonlocal coupling. $P \in[1, N / 2]$ is the number of nearest neighbors on each side of any oscillator in the ring with a coupling radius $r=\frac{P}{N}$. The environment/medium plays a crucial role in facilitating the complex collective dynamics such as decoherence, dissipation and relaxation in quantum systems [23], in coordinated rhythms in biological systems [24], and in quorum sensing 25]. The dynamics of the individual agent given by $\dot{w}_{i}$ in Eq. (1d) is related to the interactions of molecules between the cells and their environment [26 28]. In the following, we will demonstrate the existence of phase-flip chimera in an ensemble of Rössler oscillators in both periodic and chaotic regimes.

Individual Rössler oscillators in the ensemble exhibit periodic oscillations for the parameters $a=0.165, b=0.4$ and $c=8.5$. We have used random initial conditions uniformly distributed between -1 to +1 . We have fixed $N=100, k=10$, and the coupling radius $r$ as $r=0.3$ $(N=100$ is chosen only for clarity in the sense that the jumping phases can be distinctly seen. One can indeed choose any value of $N$ for our analysis). Snapshots of the instantaneous phases $\phi_{i}=\arctan \left(y_{i} / x_{i}\right), i=1,2 \ldots N$, 

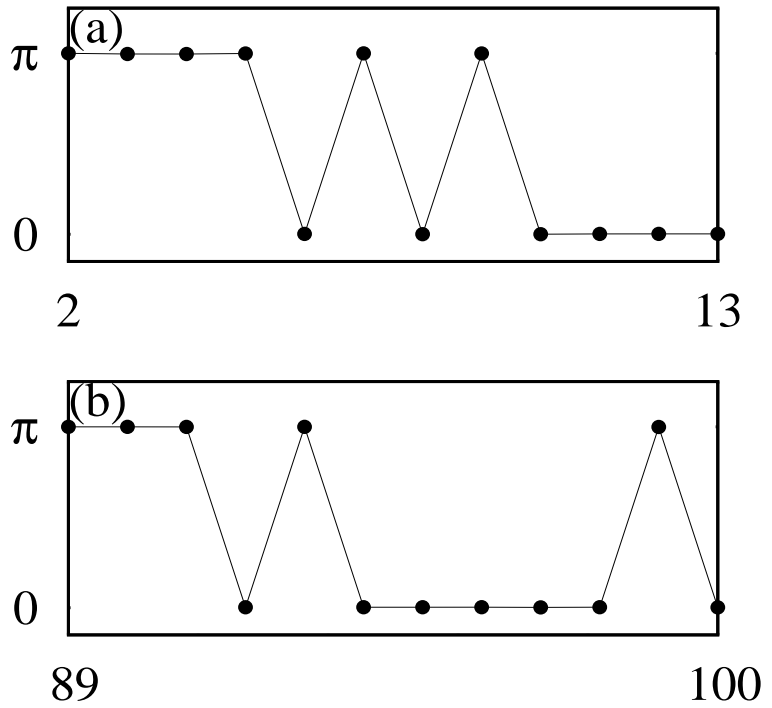

FIG. 2. (Color online) Enlarged regions of Fig. 11(g) to clearly show the phase-flip chimera characterized by coexisting coherent and incoherent domains, where the nearby oscillators exhibit in-phase oscillations in the coherent domain while the adjacent oscillators exhibit out-of-phase oscillations in the incoherent domain.

and the spatiotemporal evolution of the oscillators are depicted in Fig. 1 for different values of the strength of the nonlocal coupling $\varepsilon$. The oscillators evolve in asynchrony for $\varepsilon=0.02$ (see Figs. 1(a) and 1(b)), while their time averaged frequencies are entrained (see the inset of Fig. 1(a)). Being identical Rössler oscillators in the periodic regime, the frequencies of all the oscillators are always entrained (see the insets of Fig. 1) in the entire parameter regimes we have traced. The nonlocal coupling leads to the splitting of the ensemble into coexisting coherent and incoherent domains as depicted in Figs. 1(c) and 1(d) for $\varepsilon=0.08$, thereby confirming the existence of chimera state. Further increase in the strength of the nonlocal coupling results in the synchronous evolution of the ensemble of Rössler oscillators. Phase and complete synchronous evolution of the oscillators are clearly evident from Figs. 1(e) and 1(f), respectively, for $\varepsilon=0.5$. Phase-flip chimera emerges after the complete synchronized state, which is illustrated in Figs. 1(g) and 1(h) for $\varepsilon=1.5$. Phase-flip chimeras with two adjacent out-of-phase synchronized spatially coherent domains interspersed by a spatially incoherent domain are clearly evident from the spatiotemporal plot in Fig. 1(h), while their frequencies remain entrained. However, for the ease of visualization of the phase-flip chimera characterized by coexisting out-of-phase synchronized coherent domains (where the nearby oscillators exhibit inphase synchronized oscillations among them in each of the synchronized domains) along with an asynchronous incoherent domain (where nearby oscillators exhibit out- of-phase synchronized oscillations) interspersing the coherent domains at the phase-flip transition are enlarged and depicted in Fig. 2. It is to be noted that the chimera states investigated so far have emerged as an intermediate state in the transition from completely incoherent to completely coherent states, in general. In contrast, the phase-flip chimera emerges after the completely synchronized state. Note that these are not steady states as in the case of chimera death states [19, 20], but evolve dynamically, and they are also not two-cluster states as the oscillator index cannot be reordered.

\section{PHASE-FLIP CHIMERA IN AN ENSEMBLE OF RÖSSLER OSCILLATORS IN THE CHAOTIC REGIME}

For the parameters $a=0.1, b=0.1$ and $c=18.0$, the uncoupled Rössler oscillators evolve in (1) chaotically. Snapshots of the instantaneous phases and the spatiotemporal evolution of the oscillators are depicted in Fig. 3 for different values of coupling, $\varepsilon$. The time averaged frequencies of all the oscillators are shown in the insets of Fig. 3. One can observe that frequencies of the oscillators are entrained to the same frequency as indicated by a straight line of $w_{i}$. For lower values of $\varepsilon$, the coupled oscillators evolve in asynchrony (not shown here), whereas the ensemble of oscillators splits into coexisting coherent and incoherent domains, confirming the existence of chimera, as shown in Figs. 33(a) and 3(b) for $\varepsilon=0.5$. Increasing $\varepsilon$ further, the ensemble of oscillators evolve in complete synchrony (see Figs. 3(c) and 3(d) for $\varepsilon=1.0$ ). The synchronized oscillators become desynchronized for further larger $\varepsilon$, the dynamics of which is illustrated in the snapshot of the instantaneous phases and the spatiotemporal plots in Figs. 3(e) and 3(f), respectively, for $\varepsilon=1.2$. Phase-flip chimera emerges from the desynchronized state upon increasing the strength of the nonlocal coupling further as depicted in Figs. [3(g) and 3 (h) for $\varepsilon=2.5$.

The dynamics of the individual Rössler oscillators changes and plays a crucial role in the emergence of the observed collective dynamical behaviors of the ensemble of Rössler oscillators in Fig. 3 as a function of $\varepsilon$. The Rössler oscillators always exhibit periodic oscillations throughout the entire dynamical transition regimes of the ensemble discussed in Fig. 1. whereas in the case of chaotic oscillations of the individual oscillators the dynamical nature of the oscillators changes as will be discussed below. In the absence of the couplings $k=0$ and $\varepsilon=0$, the uncoupled individual Rössler oscillators exhibits chaotic oscillations as pointed above. A couple of uncoupled representative Rössler oscillators (namely $N=9$ and 13) exhibiting chaotic oscillations are depicted in Fig. 4(a). The filled circles and triangles in Fig. 4 are the Poincaré points. The value of $k$ in the other figures 4 (b)-(d) is fixed as $k=10$ as in Fig. 3. The ensemble of Rössler oscillators displays chimera state for 

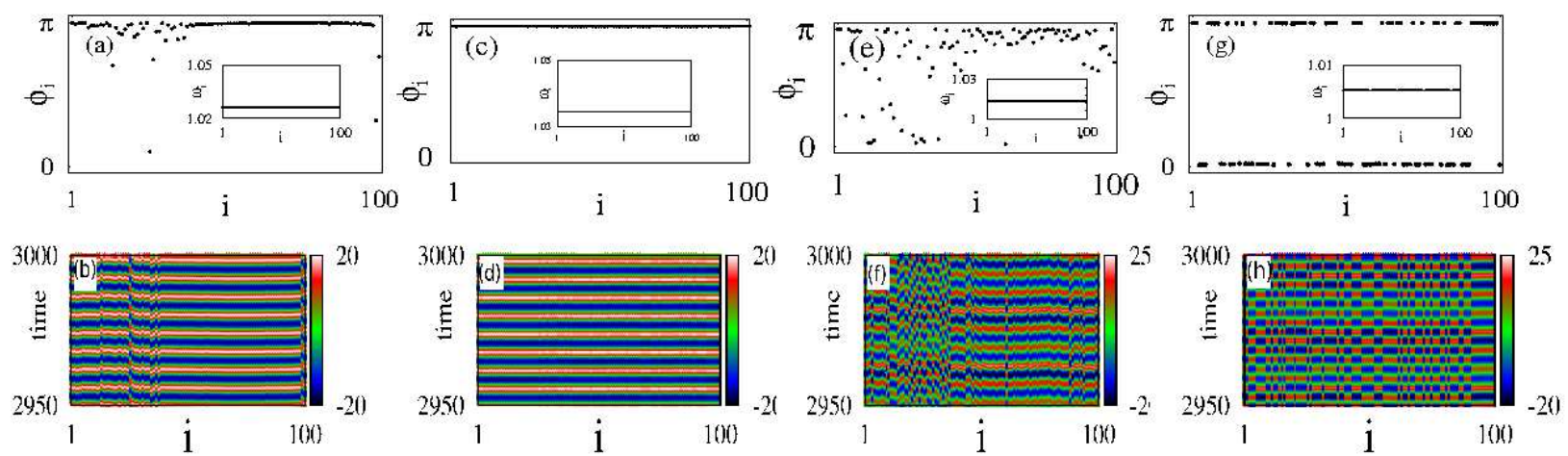

FIG. 3. (Color online) Snapshots of the instantaneous phases $\phi_{i}$ (top row) and the space-time evolution (bottom row) of the ensemble of Rössler oscillators in the chaotic regime for the coupling radius $r=0.3$ of the nonlocal coupling and for different values of the strength of the nonlocal coupling exhibiting (a)-(b) chimera state for $\varepsilon=0.5$, (c)-(d) synchronized state for $\varepsilon=1.0$, (e)-(f) desynchronized state for $\varepsilon=1.2$ and $(\mathrm{g})-(\mathrm{h})$ phase-flip chimera for $\varepsilon=2.5$. Time averaged frequencies of all the oscillators are shown in the insets. Other parameter values are $a=0.1, b=0.1, c=18$ and $\alpha=1$.
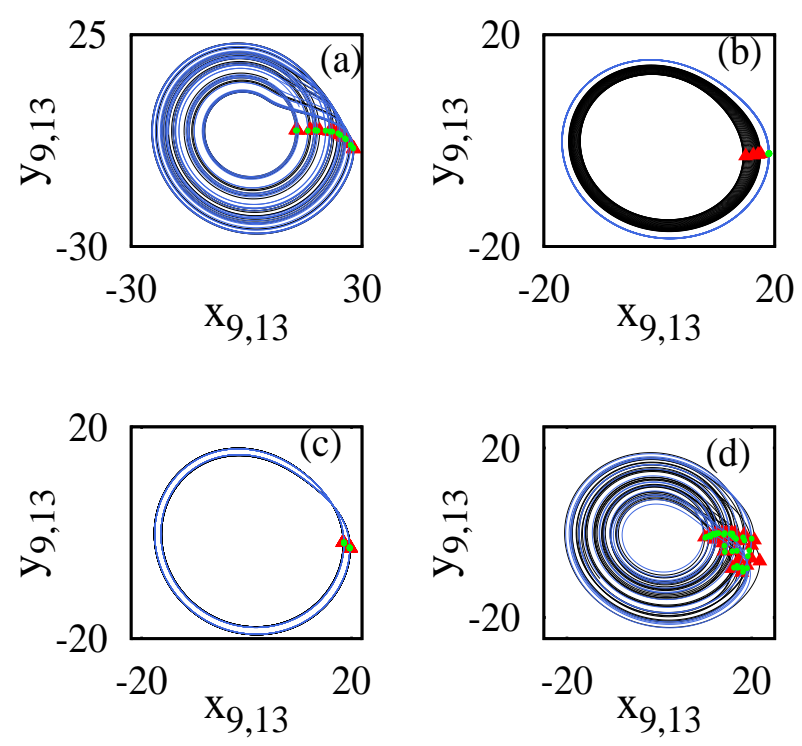

FIG. 4. (Color online) Representative class of attractors along with their Poincaré points throughout the dynamical transition of the ensemble of Rössler oscillators observed in Fig. 3 as a function of the strength of the nonlocal coupling $\varepsilon$. (a) desynchronized state for $\varepsilon=0.0$, (b) chimera state for $\varepsilon=0.5$, (c) synchronized state for $\varepsilon=1.0$, and (d) desynchronized state for $\varepsilon=1.2$. In Fig (a) the value of $k=0$, while in (c)-(d) it is fixed as 10 .

$\varepsilon=0.5$ (see Fig. 3(a)). A representative oscillator from each of the coherent and incoherent domains is displayed in Fig. 4(b). The synchronized oscillators in the coherent domain are entrained to periodic oscillations as indicated by the blue (light grey) line in Fig. 4(b), while the asynchronous oscillators in the incoherent domain exhibit chaotic oscillations as indicated by the black (dark grey) line in Fig. 4(b). The ensemble of oscillators are
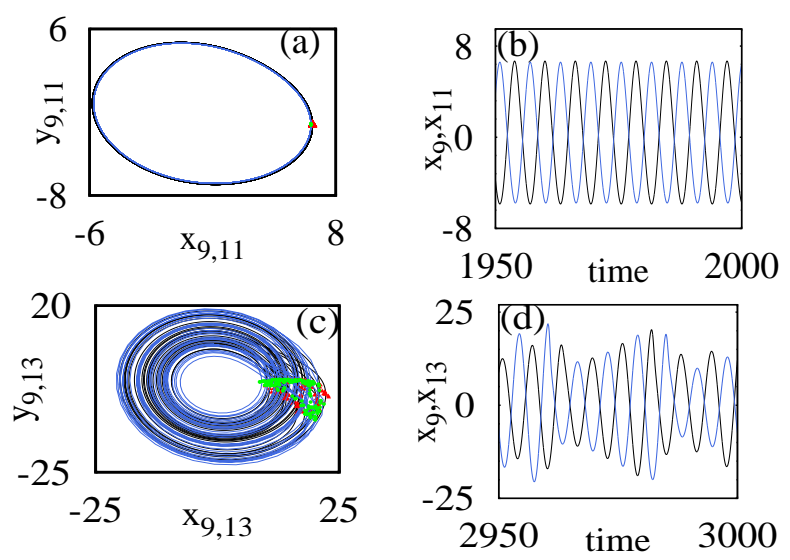

FIG. 5. (Color online) Representative class of attractors along with their Poincaré points and their time evolution plots of the out-of-phase synchronized coherent domains of the phase-flip chimera observed in Figs. 1(g) and 3(g). Top row represents the Rössler oscillators in the periodic regime and bottom row corresponds to the Rössler oscillators exhibiting chaotic oscillations.

synchronized for the strength of the nonlocal coupling $\varepsilon=1.0$ (see Fig. 3(c)), where the dynamics of the ensemble of oscillators become periodic as indicated by the representative oscillators in Fig. 4(c). The ensemble of Rössler oscillators are desynchronized as already shown in Fig. 3(e) for $\varepsilon=1.2$ rendering the oscillators to exhibit chaotic oscillations as displayed in Fig. 4(d) and the dynamics of the individual Rössler oscillators remain chaotic for further larger values of $\varepsilon$.

Attractors and the corresponding time series plots of the representative oscillators from the out-of-phase synchronized coherent domains of the phase-flip chimera are depicted in Fig. 5] The top row represents the Rössler oscillators in the periodic regime and the bottom row 
corresponds to the Rössler oscillators exhibiting chaotic oscillations. Attractors exhibting both in-phase and antiphase oscillations for the periodic case and chaotic case along with their Poincaré points are shown in Figs. [5(a) and 5 (c), respectively. Time series plots in Figs. 5 (b) and 5(d) clearly displays the out-of-phase oscillations of the two adjacent coherent domains of the phase-flip chimera.

\section{QUANTIFICATION MEASURES TO CHARACTERIZE THE CHIMERA STATES}

The notion of the strength of incoherence $S$ [17], was recently introduced by Gopal et al to characterize and to distinguish various collective dynamical states, defined as

$$
S=1-\frac{\sum_{m=1}^{M} s_{m}}{M}, s_{m}=\Theta\left(\delta-\sigma_{l}(m)\right),
$$

where $\Theta(\cdot)$ is the Heaviside step function, and $\delta$ is a predefined threshold. Normally, $\delta$ is chosen as a certain percentage value of the difference between the upper/lower bounds, $x_{l, i_{\max }} / x_{l, i_{\min }}$, of the allowed values of $x_{l, i} . M$ is the number of bins of equal size $n=N / M$. The local standard deviation $\sigma_{l}(m)$ is introduced as

$\sigma_{l}(m)=\left\langle\sqrt{\frac{1}{n} \sum_{j=n(m-1)+1}^{m n}\left[z_{l, j}-<z_{l, m}>\right]^{2}}\right\rangle_{t}, m=1,2, \ldots M$

where $z_{l, i}=x_{l, i}-x_{l, i+1}, l=1,2 \ldots d, d$ is the dimension of the individual unit in the ensemble, $i=1,2 \ldots N$, $\left.<z_{l, m}\right\rangle=\frac{1}{n} \sum_{j=n(m-1)+1}^{m n} z_{l, j}(t)$, and $\langle\ldots\rangle_{t}$ denotes the time average. When $\sigma_{l}(m)$ is less than $\delta, s_{m}=1$, otherwise $s_{m}=0$ ( $m$ in the present case is chosen as $m=20$ ). The local standard deviation $\sigma_{l}(m)$ has some finite value in the incoherent domain $\forall m$, which is always greater than $\delta$ and hence $s_{m}=0, \forall m$, thereby resulting in unit value for the strength of incoherence $S$ in the incoherent domain. On the other hand, the standard deviation $\sigma_{l}(m)$ is always zero in the coherent domain and hence $s_{m}=1, \forall m$, thereby resulting in the null value of $S$. Since the chimera states are characterized by coexisting coherent and incoherent domains, the strength of incoherence $S$ will have intermediate values between zero and one, $0<S<1$.

The strength of incoherence is shown in Figs. 6(a) and 6(b) as a function of the strength of the nonlocal coupling $\varepsilon$ characterizing the dynamical transition discussed in Figs. 1 and 3 respectively. Unit value of $S$ in the range of $\varepsilon \in(0,0.08)$ in Fig. 6(a) corroborates the asynchronous evolution of the Rössler oscillators with periodic oscillations. Intermediate value of $S$ between zero and unity confirms the existence of conventional chimera in the range of $\varepsilon \in(0.08,0.18)$. Null value of $S$ in the range of $\varepsilon \in(0.18,1.02)$ attributes to the synchronous evolution of the ensemble of Rössler oscillators. Fluctuations in the value of $S$ close to unity elucidates the existence of phase-flip chimera state. As the phase-flip chimera is
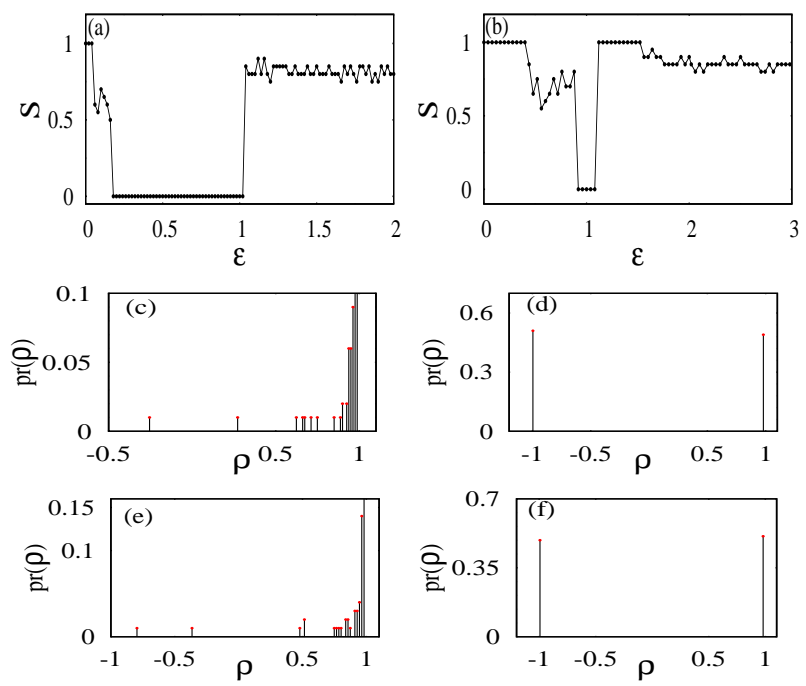

FIG. 6. (Color online) The strength of incoherence $S$ as a function of the strength of the nonlocal coupling $\varepsilon$ in (a) periodic case, (b) chaotic case. The probability distribution $\operatorname{pr}(\rho)$ of the correlation coefficient (c) conventional chimera in the periodic case (d) phase-flip chimera in the periodic case, (e) conventional chimera in the chaotic case and (f) phase-flip chimera in the chaotic case.

characterized by out-of-phase synchronized coherent domains interspersed by an incoherent domain, most of the bins during this state have a mixture of in-phase and anti-phase oscillations, while a few bins may have completely coherent (either in-phase or anti-phase) oscillations leading to fluctuating values of $S$ close to unity. Now, we will discuss the dynamical transtion observed in Fig. 3 for the chaotic oscillations of the individual Rössler oscillators in terms of the strength of incoherence $S$ displayed in Fig. 6(b). Asynchronous evolution of the oscillators are indicated by the unit value of $S$ in the range of $\varepsilon \in(0,0.48)$, whereas the intermediate value of $0<S<1$ confirms the existence of conventional chimera in the range of $\varepsilon \in(0.48,0.96)$. Narrow range of synchronized state is confirmed by $S=0$ in the range of $\varepsilon \in(0.96,1.04)$. The oscillators get desynchronized in the range of $\varepsilon \in(1.04,1.66)$ as indicated by a unit value of $S$. Fluctuations in $S$ close to unity beyond $\varepsilon=1.66$ corroborates the existence of phase-flip chimera.

We have also estimated the probability distribution of the correlation coefficient defined as

$$
\rho_{i}=\frac{\left\langle\left(x_{1}(t)-\left\langle x_{1}(t)\right\rangle\right)\left(x_{i}(t+\Delta t)-\left\langle x_{i}(t)\right\rangle\right)\right\rangle}{\sqrt{\left\langle\left(x_{1}(t)-\left\langle x_{1}(t)\right\rangle\right)^{2}\right\rangle_{t}\left\langle\left(x_{i}(t)-\left\langle x_{i}(t)\right\rangle\right)^{2}\right\rangle_{t}}},
$$

where $\mathrm{i}=1,2 . ., \mathrm{N}$, to characterize the conventional chimera and phase-flip chimera. In Eq. (4), $\langle\cdot\rangle_{t}$ represents the time average and $\Delta t$ is the time shift. The correlation coefficient is estimated by using each oscillator in the ensemble as a reference oscillator and averaging it over the number of oscillators $N$. The probability distribution of the correlation coefficient is depicted in Figs. [6(c)-[6(f) for 
different values of $\varepsilon$ in both the periodic (Figs. 6(c) and 6(d)) and chaotic (Figs. 6(e) and 6(f)) regimes. Correlation coefficient for completely synchronized oscillators acquire unit value and for antisynchronous state acquires -1 , whereas for the desynchronous state it is characterized by the intermediate values between \pm 1 . Since the conventional chimera is characterized by the coexistence of synchronized and asynchronous domains, the probability distribution of the correlation coefficient is large near unit value and small at other values of correlation coefficient as seen in Figs. 6(c) and 6(e). On the other hand, the phase-flip chimera is characterized by only two inhomogeneous states, namely in-phase and anti-phase synchronized states, the correlation coefficient of the phase-flip chimera acquires only +1 and -1 as its values. Consequently, the probability distribution of the correlation coefficient at phase-flip chimera has only two values at +1 and -1 as evident from Figs. 6(d) and 6 (f).

\section{TWO-PARAMETER PHASE DIAGRAMS}

A two-parameter phase diagram as a function of the strength of the nonlocal coupling $\varepsilon \in(0,2)$ and the coupling radius $r \in(0,0.5)$ is depicted in Fig. [7(a) to gain a global perspective of collective dynamics emerging from the ensemble of Rössler oscillators exhibiting periodic oscillations in a dynamic environment with nonlocal coupling. We have used the above quantification measures discused in Sec. IV to demarcate the different dynamical regimes of the ensemble of Rössler oscillators. The dynamical regimes delineated in Fig. 1 are indicated as $D S Y C, C H, S Y C$ and $P F C H$, which correspond to the desynchronized states, chimera states, synchronized states and phase-flip chimeras, respectively. In addition to the above regimes, we have also found the coherent states marked as $C O$, where all the oscillators evolve in coherence in the range of the coupling radius $r \in(0.05,0.2)$ and $\varepsilon \in(0.2,0.8)$.

Using the well known master stability function (MSF) formalism [33, 34], the entire parameter space in Fig. 7 can be demarcated into desynchronized state $(D S Y C)$ and synchronous state $(S Y C)$. The stability of the synchronized manifold $\left(x_{i}=x, y_{i}=y, z_{i}=z\right.$ and $w_{i}=w$; $\forall i$ ) is determined by the variational equations

$$
\begin{aligned}
& \dot{\eta}_{1 j}=-\eta_{2 j}-\eta_{3 j}, \\
& \dot{\eta}_{2 j}=\eta_{1 j}+a \eta_{2 j}, \\
& \dot{\eta}_{3 j}=\eta_{3 j}(x(t)-c)+k \eta_{4 j}+z(t) \eta_{1 j}, \\
& \dot{\eta}_{4 j}=-\alpha \eta_{4 j}+\frac{\eta_{3 j}}{2}+\varepsilon \lambda_{j} \eta_{4 j},
\end{aligned}
$$

where $x(t)$ and $z(t)$ are the solution of the uncoupled equation (11). $\eta_{i k}=\zeta_{\mathbf{i}} \mathbf{Q}_{\mathbf{k}}$, where $\zeta_{\mathbf{i}}=\left(\zeta_{\mathbf{i} 1}, \zeta_{\mathbf{i} 2}, \ldots, \zeta_{\mathbf{i N}}\right)$, and $\left(\zeta_{\mathbf{1}}, \zeta_{\mathbf{2}}, \zeta_{\mathbf{3}}, \zeta_{\mathbf{4}}\right)$ are the deviation of $(\mathbf{x}, \mathbf{y}, \mathbf{z}, \mathbf{w})$ from the synchronized solution $(x, y, z, w)$. $\mathbf{Q}_{\mathbf{k}}$ is the eigenvector of the coupling matrix $G$ and $\lambda_{j}$ are the eigenvalues,
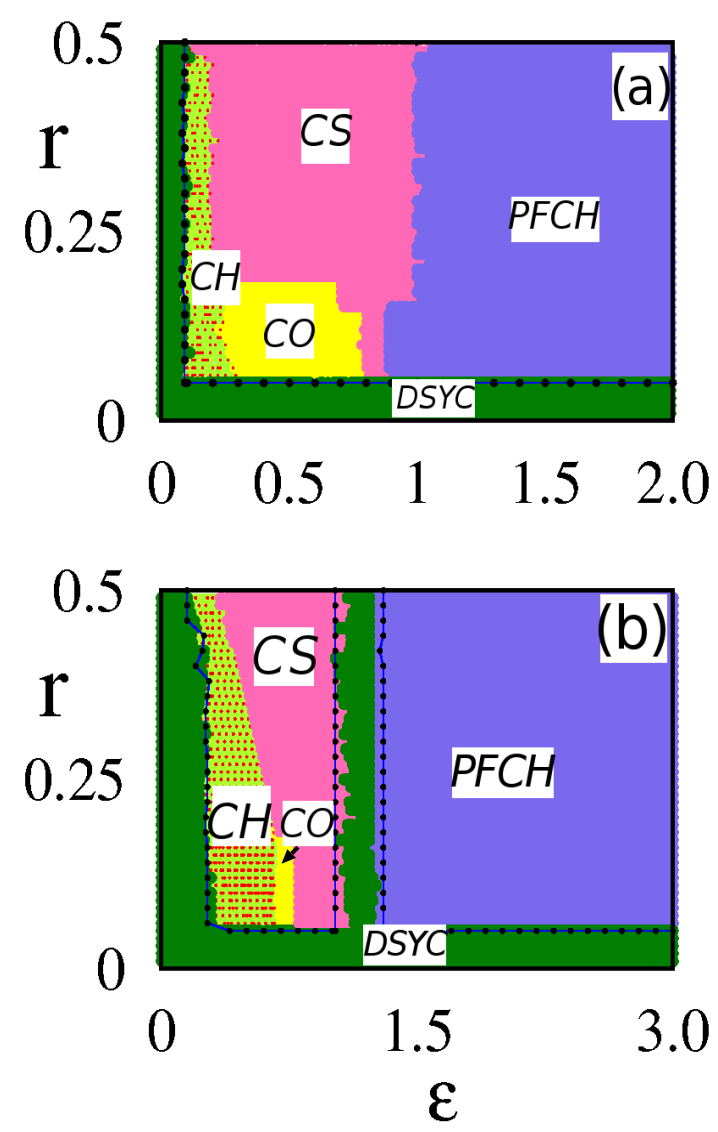

FIG. 7. (Color online) Two parameter phase diagram depicting the collective dynamical states of the ensemble of Rössler oscillators with common dynamic environment as a function of the strength of the nonlocal coupling $\varepsilon$ and the coupling radius $r$. (a) Periodic state (b) Chaotic state. The parameter spaces marked as $D S Y C, C H, P F C H, C O$, and $C S$ correspond to the desynchronized state, conventional chimera, phase-flip chimera, coherent state and complete synchronized state, respectively. The dotted lines correspond to the stability curves estimated from the eigenvalues of the variational equation (8).

where

$$
G=\left[\begin{array}{ccccc}
a_{11} & a_{12} & a_{13} & \ldots & a_{1 n} \\
a_{21} & a_{22} & a_{23} & \ldots & a_{2 n} \\
\vdots & \vdots & \vdots & \ddots & \vdots \\
a_{d 1} & a_{d 2} & a_{d 3} & \ldots & a_{d n}
\end{array}\right]
$$

with $a_{i i}=-1$ and $a_{i(i+j)}=a_{i(i-j)}=\frac{1}{2 P}$ for $i=$ $1,2, \ldots, N$ and $j=1,2, \ldots P$. Here $a_{i(N+k)}=a_{i k}$ and $a_{i(1-k)}=a_{i(N-k+1)}$ for $k=1,2, \ldots P$. The eigenvalues are given by

$$
\lambda_{j}=-1+\frac{1}{P} \sum_{k=1}^{P} \cos \left(\frac{2 \pi k}{N} j\right), j=0,1,2, \ldots, N-1 .
$$



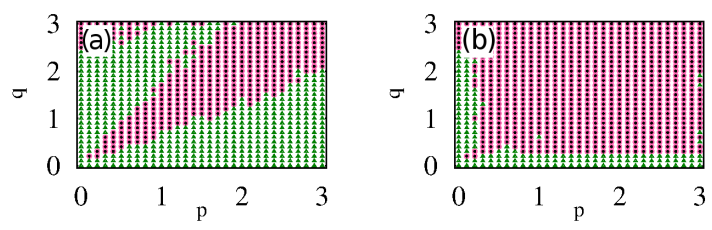

FIG. 8. (Color online) Basin of attraction depicting the coexisting phase-flip chimera (red, •) and complete synchronized states (green, $\mathbf{\Delta}$ ) in (a) periodic and (b) chaotic cases of the coupled Rössler oscillators.

The eigenvalue $\lambda_{0}$ corresponds to the perturbation parallel to the synchronization manifold, while the other $N-1$ eigenvalues correspond to the perturbation transverse to the synchronization manifold. The transverse eigenmodes should be damped out to have a stable synchronization manifold. The stability of the synchronization manifold depends only on the largest eigenvalue $\lambda_{1}=-1+\frac{1}{P} \sum_{k=1}^{P} \cos \left(\frac{2 \pi k}{N}\right)$ and the corresponding variational equation becomes

$$
\begin{aligned}
& \dot{\eta}_{11}=-\eta_{21}-\eta_{31}, \\
& \dot{\eta}_{21}=\eta_{11}+a \eta_{21} \\
& \dot{\eta}_{31}=\eta_{31}(x(t)-c)+k \eta_{41}+z(t) \eta_{11}, \\
& \dot{\eta}_{41}=-\alpha \eta_{41}+\frac{\eta_{31}}{2}-\varepsilon\left(1-\frac{1}{P} \sum_{k=1}^{P} \cos \left(\frac{2 \pi k}{N}\right)\right) \eta_{41} .
\end{aligned}
$$

Now, the value of the largest Lyapunov exponent of the variational equation (8) for each value of the parameters in the two-parameter phase diagram (Fig. 7) is used to demarcate the two-parameter phase diagram into desynchronized state $(D S Y C)$ and synchronous state $(S Y C)$ as indicated by the dotted lines in Fig. 7(a). Before the dotted lines the largest Lyapunov exponent acquires positive values while for the parameters above the dotted lines it acquires negative values attributing to the stability of the synchronization manifold. Figure 7 elucidates that the simulation results are in agreement with the results obtained using the semi-analytic approach, namely the master stability function formalism in demarcating the synchronized and desynchronized regimes. It is to be noted that the synchronous state is a multistable state with coexisting chimera states, coherent states and phase-flip chimeras depending on the distribution of the initial conditions (for a given choice of $\epsilon$ and $r$ or $\epsilon$ and $k$ ). The multistability nature of the synchronized parameter space coexisting along with the phase-flip chimera will be discussed in Fig. 8 .

A similar two-parameter phase diagram in the range of $\varepsilon \in(0,3)$ and $r \in(0,0.5]$ of the ensemble of Rössler oscillators exhibiting chaotic oscillations is shown in Fig. Z(b). The main difference between the emergent dynamics from the ensemble of Rössler oscillators with periodic oscillations (see Fig. [7(a)) and that with chaotic oscillations (see Fig. 7(b)) is that in the later case the phase-flip chimera (marked as $\mathrm{PFCH}$ ) is immediately preceded by the asynchronous oscillations, indicated by $D S Y C$, of the ensemble. That is, the dynamical transition occurs in the sequence of desynchronized state $(D S Y C)$, chimera state $(\mathrm{CH})$, coherent state $(\mathrm{CO})$ or completely synchronized state $(S Y C)$ depending on the value of $r$, again desynchronized state $(D S Y C)$ and finally phaseflip chimera $(P F C H)$ as a function of $\varepsilon$, which is evident from the two-parameter phase diagram in Fig. 7(b). The synchronized and desynchronized parameter space is also demarcated by solving the variational equation Eq. (8) as indicated by the dotted lines in Fig. 7 Here, the parameter space enclosed between the dotted lines are characterized by the negative values of the largest Lyapunov exponent of the variational equations attributing to the stable synchronized state. It is to be noted that in both the two-parameter phase diagrams, the discussed transition occurs for $r>0.05$, below which the ensemble of oscillators remain in asynchronous state elucidating that the phase-flip chimera may not emerge in the nearest neighbor coupling between the agents. It also reveals that an appropriate coupling radius is necessary for the emergence of phase-flip chimera.

In order to demonstrate the stability of the phase-flip chimera, we have depicted the basin of attraction of the ensemble, Eq. (1), in Fig. 8 as a function of $p$ and $q$, which correspond to the initial conditions of $x$ and $y$ variables. The initial conditions for the variables $z$ and $w$ are uniform random numbers distributed between -1 and 1 , whereas that of $x$ and $y$ variables are the uniform random numbers distributed between $\pm p$ and $\pm q$, respectively. It is clear that both the phase-flip chimeras (indicated by circles) and completely synchronized states (indicated by triangles) coexist for a wide choice of initial conditions corroborating the stability and robustness of the phaseflip chimera.

To explore the effect of the environmental coupling on the observed dynamical transition in detail, we have plotted the two-parameter phase diagram as a function of the strength of the nonlocal coupling $\varepsilon$ and the strength $k$ with which the local agents interact with their respective oscillators in Figs. 9(a) and 9(b) for periodic and chaotic oscillations of the individual uncoupled Rössler oscillators, respectively. The parameter space leading to desynchronous state, conventional chimera, phase-flip chimera and complete synchronous state are marked as $D S Y C, C H, P F C H$ and $C S$, respectivey, in both the figures. Asynchronous and completely synchronous parameter space are also demaracted (indicated by dotted lines) using the value of the largest Lyapunov exponent of the variational equation (8). In the periodic regime of the Rössler oscillators, the coupled oscillators remain asynchronous in the entire range of $\varepsilon$ for small values of $k$ as seen in Fig. 9(a). In the range of $k \in(2.1,5.6)$, desynchronized state is followed by conventional chimera and the phase-flip chimera as $\varepsilon$ is increased. Increasing $k$ further, the conventional chimera and phase-flip chimera regimes are seperated by complete synchronized regime upto to $k=12.6$. Transition from asynchronous to com- 

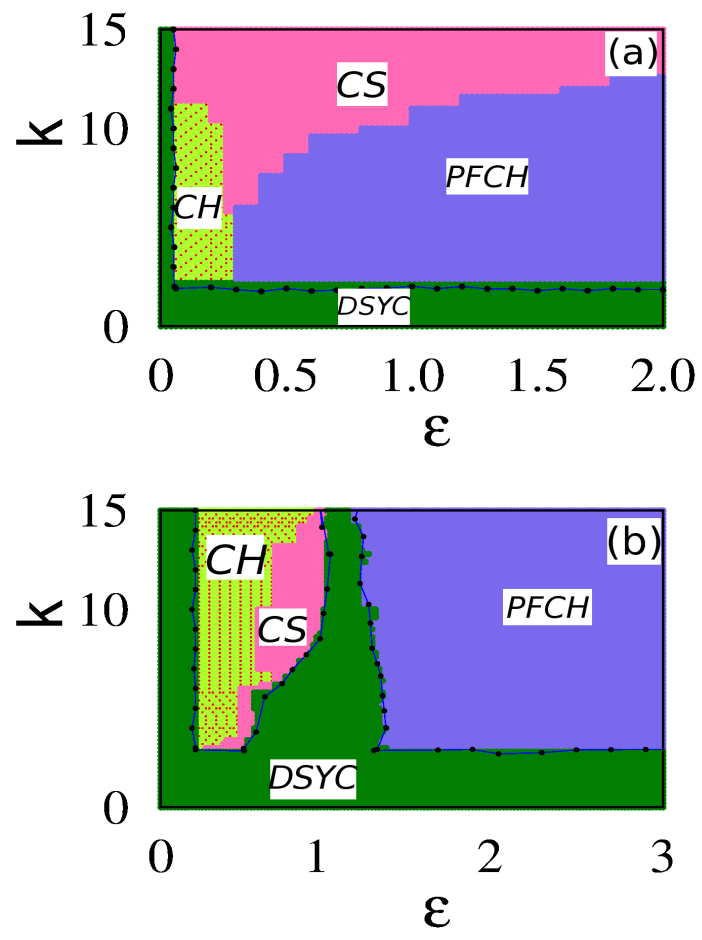

FIG. 9. (Color online) Two parameter phase diagram depicting the collective dynamical states of the ensemble of Rössler oscillators with common dynamic environment as a function of $k$ and $\varepsilon$. The parameter space marked as $D S Y C, C H, P F C H$ and $C S$ corresponds to the desynchronized state, conventional chimera, phase-flip chimera, and complete synchronized state, respectively. The dotted lines corresponds to the stability curves estimated from the eigenvalues of the variational equation (8).

plete synchronized state occurs for $k>12.6$ as a function of $\varepsilon$. Thus the emergence of phase-flip chimera is spread over a wide range of $k \in(2.1,12.6)$ and $\varepsilon$. For the chaotic oscillations of the individual Rössler oscillators, we have observed similar transitions as in Fig. 9(a) as a function of $k$ and $\varepsilon$ except for the fact that the range of phase-flip chimera extends to much wider range of $k$ and $\varepsilon$ (See Fig. 9(b)) while the desynchronized state preceeds the phase-flip chimera.

The existence of phase-flip bifurcation/transition in two coupled oscillators induced by the environmental coupling in Eq. (11) was shown in [27, 28]. We find that the environmental coupling among the agents and the oscillators induces phase-flip transition/bifurcation while the nonlocal coupling among the agents in the common environment facilitates the onset of incoherent domain at the phase-flip transition. In the absence (or for low values of the strength) of the environmental coupling one cannot observe the phase-flip chimera as is evident from the two-phase diagram in Fig. 9 We have also found that the emergence of the phase-flip chimera depends on the relaxation time of the environmental coupling in
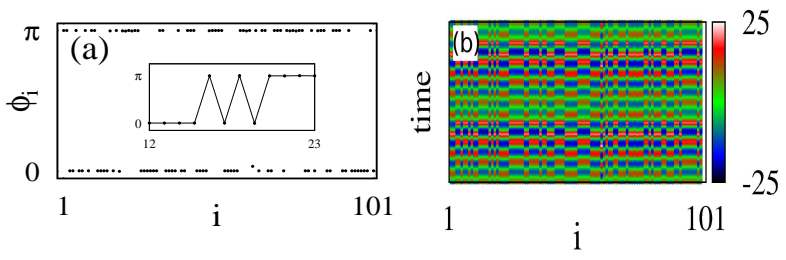

FIG. 10. (Color online) (a) Snapshots of the instantaneous phases $\phi_{i}$ and (b) the space-time evolution of the ensemble of Rössler oscillators in the chaotic regime with global coupling between the agents for the value of the strength of the global coupling $\varepsilon=2.5$.

Eq. 1(d) and phase-flip chimera emerges for the parameter $\alpha \geq 1$, which determines the relaxation time of the external agent $w_{i}$ (See Appendix A for more details).

\section{PHASE-FLIP CHIMERA WITH GLOBALLY COUPLED AGENTS}

It is also to be noted that we have identified the emergence of the phase-flip chimera in the case of globally coupled agents. Snapshots of the instantaneous phases $\phi_{i}$ and the space-time evolution of the ensemble of Rössler oscillators in the chaotic regime but with global coupling between the agents for the value of the strength of the global coupling $\varepsilon=2.5$ is shown in Fig. 10. The value of the other parameters and the distribution of the initial conditions are the same as in Fig. 3. It is clear from the figure that the phase-flip chimera characterized by two adjacent out-of-phase synchronized coherent domains, where nearby oscillators are exhibiting in-phase synchronized oscillations, interspersed by an incoherent domain, where the nearby oscillators exhibit out-of-phase oscillations (see the inset of Fig. 10), exists even with the global coupling between the agents in the common environment. We have also confirmed the emergence of phase-flip chimera from the ensemble for the periodic oscillations of the individual Rössler oscillators with the global coupling between the agents. It is known that in an ensemble of globally coupled oscillators one can reorder the spatial index of the oscillators such that the spatial inhomogenity with coexisting in-phase and out-of-phase synchronized oscillators in the phase-flip chimera state (see Fig. 2) can be recasted as a two-cluster state 35 37. However, we would like to point out that once the spatial ordering of the globally coupled oscillators are fixed by indexing them from 1 to $N$, then the distribution of initial conditions as stated above among the oscillators results in spatially inhomogeneous states with coherent out-of-phase synchronized domains interspersed by incoherent domain comprised of nearby oscillators exhibiting in-phase and anti-phase oscillations. This confirms the emergence of phase-flip chimera even with global coupling among the agents. In- 
deed, several recent investigations reported the existence of chimera states in an ensemble of globally coupled oscillators [17, 18, 29 32].

Finally, we have also checked whether phase-flip chimeras occur when the coupling is additionally given to $x$ or $y$ variables that is to Eq. (1a) or Eq. (1b). In these cases only transition from desynchronized state to synchronized state occurs (see Appendix B for more details).

\section{CONCLUSIONS}

In summary, we have identified an interesting type of a collective dynamical regime, called phase-flip chimeras, in an ensemble of identical Rössler oscillators coupled indirectly via the agents from a common dynamic environment, where the agents are coupled nonlocally with a coupling radius $r$. Such interactions are found in the diffusion of biomolecues between the cells and their environment [26]. The phase-flip chimera is characterized by two out-of-phase synchronized spatially coherent domains interspersed by a spatially incoherent domain comprised of nearby oscillators exhibiting out-ofphase oscillations. The oscillators in each of the coherent domains exhibit phase synchronized oscillations among themselves, whereas the two adjacent coherent domains in the phase-flip chimera exhibit out-of-phase synchronized oscillations. The robustness of the phaseflip chimera is also confirmed by depicting its occurrence in a wide range of parameters using the two-parameter phase diagram. Further, it is also shown that the phaseflip chimera is preceded by the conventional chimera and it emerges only after the completely synchronized state emerges. In the chaotic regime the phase-flip chimera is immediately preceded by asynchronous state, which in turn emerges as a desynchronized state from the synchronous evolution of the ensemble of oscillators. We have used the strength of incoherence, probability distribution of the correlation coeffecient and the master stability function to characterize the observed dynamical transition of the ensemble of the Rössler oscillators. It is also confirmed that the phase-flip chimera emerges even in the global coupling among the agents, whereas with the nearest neighbor coupling such a state does not emerge.

\section{ACKNOWLEDGMENTS}

The work of $\mathrm{VKC}$ forms part of a research project sponsored by INSA Young Scientist Project under Grant No. SP/YSP/96/2014. DVS is supported by the SERBDST Fast Track scheme for young scientist under Grant No. ST/FTP/PS-119/2013. ML is supported by a NASI Platinum Jubilee Senior Scientist Fellowship.
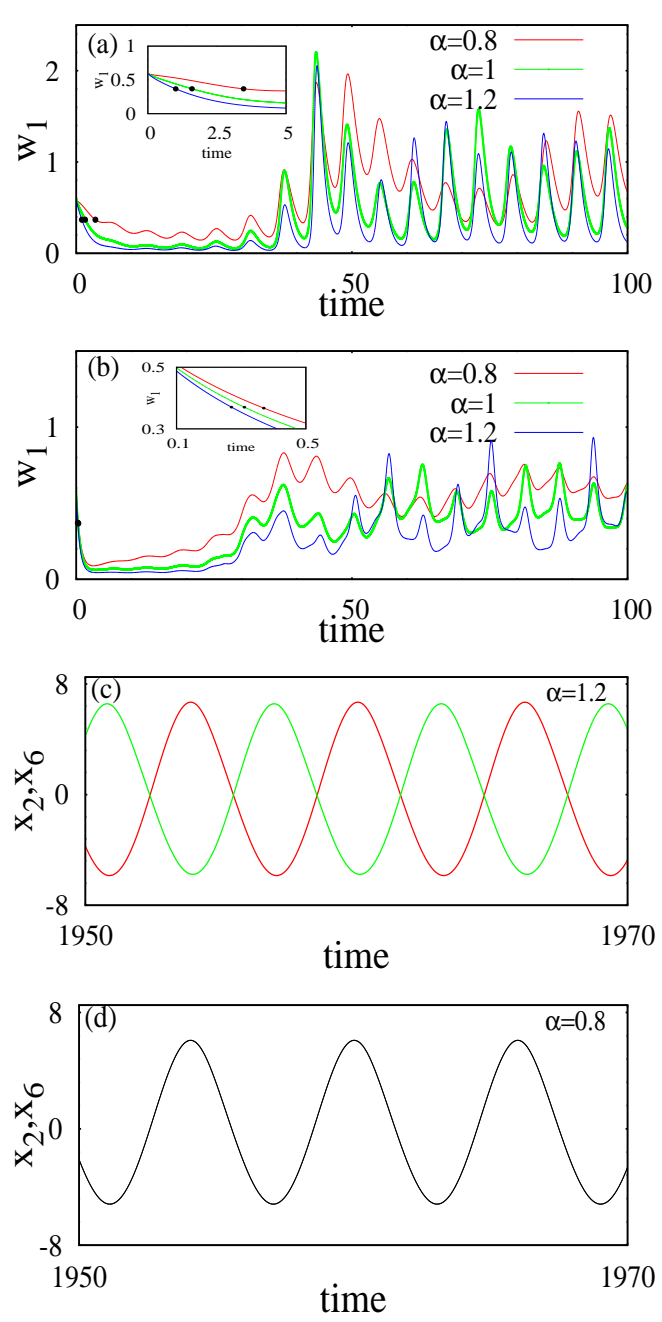

FIG. 11. (Color online) Dynamics of the agent $w_{1}$ is shown in (a) for $\varepsilon=0$, and (b) for $\varepsilon=1.5$. In both the figures $k=10$. The dynamics of random oscillators are shown in (c) and (d) for different values of the relaxation parameter $\alpha$.

\section{APPENDIX A: RELAXATION TIME OF THE ENVIRONMENTAL COUPLING EQ. (1d)}

The dependence of the dynamics of the oscillators on the relaxation time is depicted in Fig. 11. Dynamics of the agent $w_{1}$ (as an example) is shown in Fig. 11(a) for $\varepsilon=0$, and in Fig. 11)(b) for $\varepsilon=1.5$. The dynamics of randomly chosen oscillators are shown in Figs. 11(c) and 11(d) for different values of the relaxation parameter $\alpha$ occurring in Eq. (1d).

As the value of the parameter $\alpha$ is increased the relaxation time (pointed out by filled circles in the insets) decreases both in the absence (Fig. 11(a)) and in the presence (Fig. 11(b)) of the coupling between the agents $w_{i}$ in Eq. (1d). It is to be noted that the relaxation time is very less in the presence of the coupling between the oscillators (See Fig. 11(b)) compared to the uncoupled oscillators (See Fig. 11(a)). Further, the value of the pa- 

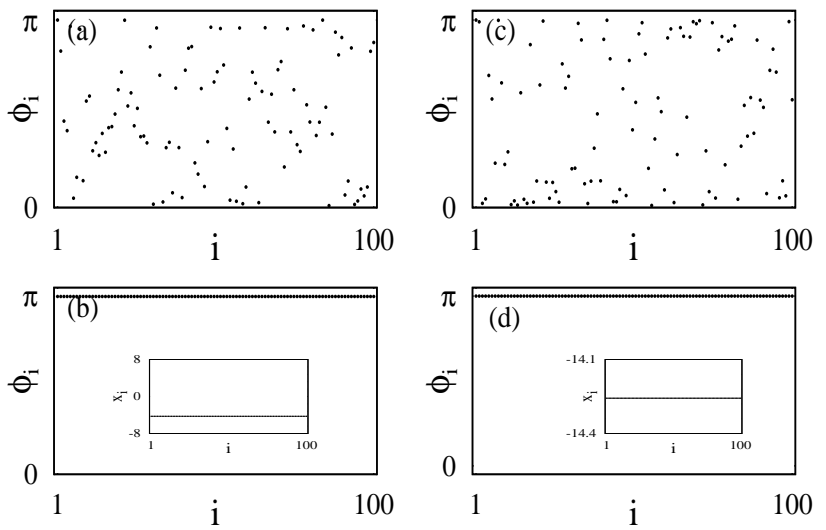

FIG. 12. (Color online) Snapshots of the instantaneous phases $\phi_{i}$ of the ensemble of Rössler oscillators coupled via $y$-variable displaying (a) desynchronized state for $\varepsilon=0.005$, (b) completely synchronized state for $\varepsilon=0.02$, (c) desynchronized state for $\varepsilon=0.005$ and (d) completely synchronized state for $\varepsilon=0.04$. Left column for periodic oscillations and right column for chaotic oscillations of the Rössler oscillators. Insets in Fig.(b) and (d) depict the snapshots of $x_{i}$.

rameter $\alpha$ which determines the relaxation time dictates the collective behavior of the ensemble of the oscillators. Phase-flip chimeras are observed for $\alpha \geq 1$. For $\alpha=1$ the phase-flip chimera is already shown in Figs. 1 and 3. For $\alpha>1.2$, the out-of-phase oscillations of the adjacent outof-phase synchronized coherent domains of the phase-flip chimera are represented by a couple of representative oscillators in Fig. 11(c). On the other hand, for $\alpha<1.0$, we observe only synchronized state for any choice of initial conditions and coupling strength as shown by the representative oscillators in Fig. 111(d).
Thus we find that the emergence of the phase-flip chimera depends on the relaxation time of the environmental coupling in Eq. (1d) and that it emerges for the parameter $\alpha \geq 1$, which determines the relaxation time of the $w_{i}$ 's.

\section{APPENDIX B: DYNAMICS OF COUPLING WITH OTHER VARIABLES}

We have also examined the typical transition from desynchronized state to synchronized states by coupling the agent $w_{i}$ to the other variables $x_{i}$ or $y_{i}$ in the same fashion as in Eq. (1d). Snapshots of the instantaneous phases $\phi_{i}$ of the ensemble of Rössler oscillators coupled via the $y$-variable are shown in Fig. 12, The left column corresponds to the case of periodic oscillations when the oscillators are uncoupled, while the right column corresponds to the case of the chaotic oscillations of the uncoupled oscillators. Desynchronized state is observed for $\varepsilon=0.005$ (See Fig. 12(a)) for the case of periodic oscillations. Upon increasing the coupling strength the oscillators are completely synchronized as shown in Fig. 12(b) for $\varepsilon=0.02$ and remains synchronized for any large coupling strength. For the case of chaotic oscillations of the uncoupled Rössler oscillators, the oscillators are desynchronized for low values of the coupling strength as shown in Fig. 12(c) for $\varepsilon=0.005$ and remain completely synchronized above a threshold value of $\varepsilon$ as illustrated in Fig. 12(d) for $\varepsilon=0.04$. Hence, only the existence of completely synchronized state from the desynchronized state is found and we are not able to find phase-flip chimera for any value of $k$ and $\varepsilon$. Similar scenario arises for $x$ coupling as well (which we do not present here).
[1] Y. Kuramoto, Chemical Oscillations, Waves, and Turbulence, (Springer-Verlag., Berlin, 1984).

[2] A. Pikovsky, M. Rosenblum, and J. Kurths, Synchronization A Universal Concept in Nonlinear Sciences (Cambridge University Press, Cambridge, England, 2001).

[3] A. T. Winfree, The Geometry of Biological Time (Springer, New York, 2001).

[4] D. Battogtokh and Y. Kuramoto, Nonlinear. Phenom. Complex. Sys. 5,380 (2002); D. M. Abrams and S. H. Strogatz Phys. Rev. Lett. 93, 174102 (2004); D. M. Abrams, R. Mirollo, S. H. Strogatz and D. A. Wiley, Phys. Rev. Lett. 101, 084103 (2008); G. C. Sethia, A. Sen and F. M. Atay, Phys. Rev. Lett. 100, 144102 (2008); M. J. Panaggio and D. M. Abrams, Nonlinearity 28, R67 (2015).

[5] C. .R. Laing and C. C. Chow, Neural Computation 13, 1473 (2000); C. R. Laing, Phys. Rev. E 81, 066221 (2010); G. Bordyugov, A. Pikovsky, and M. Rosenblum, Phys. Rev. E 82, 035205 (2010); S. Olmi, A. Politi, and A. Torcini, Europhys. Lett.92,60007 (2010).
[6] I. Omelchenko, Y. Maistrenko, P. Hövel, and E. Schöll, Phys. Rev. Lett. 106, 234102 (2011); I. Omelchenko, B. Riemenschneider, P. Hövel, Y. Maistrenko, and E. Schöll, Phys. Rev. E 85, 026212 (2012); I. Omelchenko, O. E. Omelchenko, P. Hövel, and E. Schöll, Phys. Rev. Lett. 110, 224101 (2013).

[7] Jane H. Sheeba, V. K. Chandrasekar and M. Lakshmanan, Phys. Rev. E 79, 055203 (4 pp) (R) (2009);Phys. Rev. E 81, 046203 (10 pp) (2010).

[8] S. R. Ujjwal, and R. Ramaswamy, Phys. Rev. E 88, 032902 (2013); D. Pazo, and E. Montbrió, Phys. Rev. X, 4, 011009, (2014); L. Schmidt, K. Schönleber, K. Krischer, and V. Garci'aMorales, Chaos 24, 013102 (2014).

[9] V. K. Chandrasekar, R. Suresh D.V. Senthilkumar and M. Lakshmanan, Euro.Phys Letters111 6008 (2015); Bidesh K. Bera, Dibakar Ghosh, M. Lakshmanan, Phys. Rev E 93, 012205 (2015).

[10] M. R. Tinsley, S.Nkomo and S. Showalter, Nat. Phys. 8, 662 (2012); S. Nkomo, M. R. Tinsley, and K. Showalter, Phys. Rev. Lett. 110, 244102 (2013). 
[11] A. M. Hagerstrom, T. E. Murphy, R. Roy, P. Hövel, I. Omelchenko, and E. Schöll, Nature Phys. 8, 658661, (2012).

[12] E. A. Martens, S. Thutupalli, A. Fourriére and O. Hallatschek, Proc. Nat. Acad. Sci. USA, 110, 1056310567 (2013).

[13] G. Filatrella, A. H. Nielsen, and N. F. Pedersen, Eur. Phys. J. B 61 (4),485 (2008).

[14] N. C. Rottenberg, C. J. Amlaner, and S. L. Lima, Neurosci Biobehav Rev. 24,817 (2000).

[15] E. Olbrich, J. C. Claussen, and P. Achermann, Phil.Trans.R. Soc. A 369,3884 (2011)

[16] G. C. Sethia, A. Sen, and G. L. Johnston, Phys. Rev. E. 88, 042917 (2013).

[17] V. K. Chandrasekar, R. Gopal, A. Venkatesan, and M. Lakshmanan, Phys. Rev. E. 90, 062913 (2014).

[18] G. C. Sethia and A. Sen, Phys. Rev. Lett. 112, 144101 (2014); A. Yeldesbay, A. Pikovsky, and M. Rosenblum, Phys. Rev. Lett. 112, 144103 (2014).

[19] A. Zakharova,M. Kapeller and E. Schöll Phys. Rev. Lett 112, 154101 (2014); A. Zakharova, M. Kapeller, and E. Schöll, J. Phys. Conf.Series (2015), arXiv 1503.03371;

[20] K. Premalatha, V. K. Chandrasekar, M. Senthilvelan and M. Lakshmanan, Phys. Rev. E 91, 052915 (2015) arXiv:1511.07220 (Phys. Re. E (2016) to appear).

[21] N. Semenova, A. Zakharova, V. Anishchenko, and E. Schöll, arXiv:1512.07036r1 (2015).

[22] J. M. Cruz, J. Escalona, P. Parmananda, R. Karnatak, A. Prasad, and R. Ramaswamy, Phys. Rev. E 81, 046213 (2010); A. Prasad, J. Kurths, S. K.Dana and R. Ramaswamy, Phys. Rev. E 74, 035204(2006);A. Sharma, M. D. Shrimali, A. Prasad, R. Ramaswamy and U. Feudel, Phys. Rev. E 84, 016226 (2011).

[23] H. Ammann, R. Gray, I. Shvarchuck, and N. Christensen, Phys. Rev. Lett. 80, 4111 (1998); W. H. Zurek, S. Habib, and J. P. Paz, Phys. Rev. Lett. 70, 1187 (1993); S. A. Gurvitz, L. Fedichkin, D. Mozyrsky, and G. P. Berman, Phys. Rev. Lett. 91, 066801 (2003); S. Braig and K. Flensberg, Phys. Rev. B 68, 205324 (2003).
[24] A. Kuznetsov, M. Krn, and N. Kopell, SIAM J. Appl. Math.65, 392 (2004); R. Wang and L. Chen, J. Biol. Rhythms20, 257 (2005); D. Gonze, S. Bernard, C. Waltermann, A. Kramer, and H. Herzel, Biophys. J.89, 120 (2005).

[25] B. W. Li, C. Fu, H. Zhang and X. Wang, Phys. Rev. E 86,046207 (2012).

[26] E. Ullner, A. Zaikin, E. I. Volkov, and J. Garcia-Ojalvo, Phys. Rev. Lett. 99, 148103 (2007); E. Ullner, A. Koseska, J. Kurths, E. Volkov, H. Kantz, and J. GarciaOjalvo, Phys. Rev. E. 78, 031904 (2008).

[27] V. Resmi, G. Ambika, and R. E. Amritkar, Phys. Rev. E 81, 046216 (2010).

[28] A. Sharma, M. D. Shrimali and S. K. Dana, Chaos 22, 023147 (2012).

[29] T. Banerjee and D. Ghosh, Phys. Rev. E 89, 052912 (2014).

[30] L. Schmidt and K. Krischer, Phys. Rev. Lett. 114, 034101 (2015)

[31] L. Schmidt and K. Krischer, Chaos 25, 064401 (2015).

[32] M. I. Bolotov, G. V. Osipov, and A. Pikovsky, Phys. Rev. E 93, 032202 (2016).

[33] L. M. Pecora and T. L. Carroll, Phys. Rev. Lett. 80, 2109 (1998).

[34] S. Acharyya and R. E. Amritkar, Phys. Rev. E 92, 052902 (2015).

[35] S. C. Manrubia, A.S. Mikfailov, O. D. Zanatte Emenrgence of Dynamical Orer: Synchronization Phenomena in Complex Systems (World Scirtific, Singapore, 2004).

[36] K. Okuda, Physica D 63, 424 (1993); V. Hakim and W.J. Rappel, Phys. Rev. A 46, R7347 (1992); N. Nakagawa and Y. Kuramoto, Prog. Theor. Phys. 89, 313 (1993); Y. Kuramoto, Prog. Theor. Phys.94, 321 (1995).

[37] D. H. Zanette and A. S. Mikhailov, Phys. Rev. E 57, 276 (1998); D. H. Zanette and A. S. Mikhailov, Phys. Rev. E 62, R7571(R) (2000);W. Wang, I. Z. Kiss, and J. L. Hudson, Chaos 10, 248 (2000); M. Wickramasinghe and I. Z. Kiss, PLoS ONE 8, e80586 (2013). 